\title{
Comparison of fabric skins for the simulation of sweating on thermal manikins
}

\author{
Barbara Koelblen $^{1,2}$ - Agnes Psikuta ${ }^{2}$ Anna Bogdan ${ }^{1} \cdot$ Simon Annaheim ${ }^{2}$. \\ René M. Rossi ${ }^{2}$
}

Received: 29 August 2016 /Revised: 8 February 2017 / Accepted: 1 March 2017 /Published online: 16 March 2017

(C) ISB 2017

\begin{abstract}
Sweating is an important thermoregulatory process helping to dissipate heat and, thus, to prevent overheating of the human body. Simulations of human thermo-physiological responses in hot conditions or during exercising are helpful for assessing heat stress; however, realistic sweating simulation and evaporative cooling is needed. To this end, thermal manikins dressed with a tight fabric skin can be used, and the properties of this skin should help human-like sweat evaporation simulation. Four fabrics, i.e., cotton with elastane, polyester, polyamide with elastane, and a skin provided by a manikin manufacturer (Thermetrics) were compared in this study. The moisture management properties of the fabrics have been investigated in basic tests with regard to all phases of sweating relevant for simulating human thermo-physiological responses, namely, onset of sweating, fully developed sweating, and drying. The suitability of the fabrics for standard tests, such as clothing evaporative resistance measurements, was evaluated based on tests corresponding to the middle phase of sweating. Simulations with a head manikin coupled to a thermo-physiological model were performed to evaluate the overall performance of the skins. The results of the study showed that three out of four evaluated fabrics have adequate moisture management properties with regard to the simulation of sweating, which was confirmed in the coupled simulation with the head manikin. The presented tests are helpful for
\end{abstract}

Agnes Psikuta

agnes.psikuta@empa.ch

1 Air-Conditioning and Heating Department, Warsaw University of Technology, Warsaw, Poland

2 Laboratory for Biomimetic Membranes and Textiles, Empa, Swiss Federal Laboratories for Materials Science and Technology, Lerchenfeldstrasse 5, 9014 St. Gallen, Switzerland comparing the efficiency of different fabrics to simulate sweat-induced evaporative cooling on thermal manikins.

Keywords Fabric skin · Thermal manikin ·

Thermo-physiological simulator $\cdot$ Sweating simulation .

Evaporative resistance

\section{Introduction}

Sweating is one of the thermoregulatory processes within the human body which is especially important in hot conditions or while exercising. Sweat evaporation contributes to body cooling and preventing the increase of core temperature above values leading to hyperthermia or even heat stroke. Therefore, the heat and mass transfer resulting from sweating is crucial while assessing heat stress.

In nowadays research, sweat-induced evaporative cooling is often simulated with the use of thermal manikins in order to avoid drawbacks of human subject studies such as ethical issues, inter- and intra-subject variability, and high costs. Different sweating simulation methods are applied, such as the following:

- Pre-wetted tight fabric skin put on a dry thermal manikin, e.g., "Tore" (Kuklane et al. 2006)

- Water-filled manikin with a waterproof but vaporpermeable surface, e.g., "Walter" (Fan and Chen 2002)

- Manikin with an inner skin spreading water superficially and an outer vapor-permeable skin, e.g., "Coppelius" (Meinander 1997)

- Manikin with a porous metal surface with superficial sweating, e.g., advanced automotive manikin "ADAM" (Burke and McGuffin 2001) 
- Manikin with a supply of water to a fabric skin by means of sweating outlets distributed over the manikin's surface, e.g., sweating agile manikin "SAM" (Richards and Mattle 2001) and "Newton" (Thermetrics, Seattle, USA)

Phenomena related to sweat evaporation are important in standard tests, such as clothing evaporative resistance $\left(R_{\mathrm{ET}}\right)$ measurements, e.g., on a sweating guarded hot plate (ISO 1993) and for the evaluation of human thermo-physiological responses to given conditions. Thermal manikin measurements of $R_{\mathrm{ET}}$ allow a realistic distribution of the contact area and the air gap between the manikin and the clothing as well as between the layers of garments. According to the test standard (ASTM 2015), all aforementioned sweating simulation methods can be applied. For the assessment of human thermo-physiological responses in hot conditions or for high metabolic rate, a thermal sweating manikin coupled with a mathematical model of human physiology can be used, i.e., a thermo-physiological simulator (e.g., Psikuta et al. 2008; Psikuta et al. 2014). The model and the thermal manikin exchange data in a real-time feedback loop, enabling thermophysiological predictions based on the real conditions of the experiment, such as environmental parameters and clothing. Thermo-physiological simulators can be useful in many research fields, e.g., clothing research, medical applications, car industry, and indoor thermal comfort research (e.g., Rugh et al. 2004; Foda and Siren 2012; Psikuta et al. 2013; Martínez et al. 2017). The sweating simulation method has to allow dynamic changes of sweat rate; therefore, only manikins with varying water supply rate are used for such coupling (Psikuta et al. 2016).

Until now, the phenomena related to sweat secretion and evaporation have been investigated mostly with regard to standard tests, and the main issues that have been reported in the literature were the following:

- The discrepancy between manikin surface and wet skin surface temperature (Ueno and Sawada 2012; Wang et al. 2010a; Wang et al. 2012; Wang et al. 2010b)

- Different results obtained using the heat loss and mass loss methods (Havenith et al. 2008; Wang et al. 2011) which are both recommended in the standard (ASTM 2015)

- The effect of applying different sweat rates for measurements performed with a constant supply of water to the fabric skin (Lu et al. 2016a)

- The discrepancies associated with the sweating simulation method (Lu et al. 2016b; Wang et al. 2009)

The aforementioned studies mostly focused on tests with a fully wetted skin as the measurements of $R_{\mathrm{ET}}$ have to be performed in a saturated state of the fabric and in steady-state conditions. For the simulation of human thermophysiological responses, however, an investigation of phenomena occurring in all phases of sweating is needed, such as the onset of sweating, the fully developed sweating, and the drying phase.

Similarly, the desired properties of the fabric skin for sweating simulations are not explicit and ASTM (2015) does not refer to any standard skin for the measurement of $R_{\mathrm{ET}}$. The properties of different fabrics with regard to $R_{\mathrm{ET}}$ measurements have been previously investigated for three fabrics, i.e., cotton, blend of cotton and polyester, and polyester (Wang et al. 2015). However, relevant information about the sweating simulation protocol has not been reported, which hinders the interpretation of the results from this study. Additionally, the study focused on measurements with a fully wetted skin and, therefore, all of the fabrics' properties relevant for thermo-physiological transient simulations with wetting and drying phases were not addressed.

In this paper, a detailed study of the moisture management properties of four fabrics typically used as skin has been presented. Basic tests have been conducted to assess the performance of the fabrics in different phases of sweating, i.e., the onset of sweating, fully developed sweating and drying, with the middle phase corresponding to standard $R_{\mathrm{ET}}$ measurement. Additionally, measurements with a head manikin coupled to a thermo-physiological model have been conducted in order to evaluate the overall performance of the skins in realistic conditions, including the anatomical shape and the moisture migration phenomena that may influence the sweating simulation.

\section{Methods}

\section{Fabrics}

Four fabrics were compared to assess their suitability for use as skin for thermal sweating devices, namely, cotton with elastane $(\mathrm{CO})$, polyester (PE), polyamide with elastane (PA), and the skin provided by a manikin manufacturer (Thermetrics, Seattle, USA), referred to as Thermetrics' skin (TH) composed of polyamide and elastane. Cotton and polyamide are used as skins in various research laboratories, the Thermetrics' skin is provided by the manufacturer with the Newton-type manikins, and polyester showed some high wicking properties and was, therefore, selected as a reference fabric. The basic characteristics of the fabrics are listed in Table 1.

Prior to all tests, the fabrics were washed in tap water with soap, rinsed abundantly, and dried, in order to ensure that all samples would be in the same state before the measurements.

\section{Experimental design}

The evaluation of skins for the simulation of human thermophysiological responses consisted of measurements addressing three phases of sweating, namely, the onset of sweating, 
Table 1 Basic characteristics of the tested fabrics

\begin{tabular}{lllll}
\hline Name & Thickness $[\mathrm{mm}]$ & $\begin{array}{l}\text { Mass per unit } \\
\text { area }\left[\mathrm{g} / \mathrm{m}^{2}\right]\end{array}$ & $\begin{array}{l}\text { Fabric's } \\
\text { composition }\end{array}$ & Manufacturer \\
\hline $\begin{array}{l}\mathrm{CO} \\
\text { cotton with elastane }\end{array}$ & $0.92 \pm 0.03$ & $208 \pm 1$ & $95 \mathrm{CO} / 5 \mathrm{EL}$ & Unknown \\
$\begin{array}{l}\text { PE } \\
\text { polyester }\end{array}$ & $0.70 \pm 0.01$ & $155 \pm 1$ & $100 \mathrm{PE}$ & Comfortrust AG, Switzerland \\
$\begin{array}{l}\text { TH } \\
\begin{array}{l}\text { Thermetrics' skin } \\
\text { PA } \\
\text { polyamide with elastane }\end{array}\end{array}$ & $0.66 \pm 0.01$ & $195 \pm 1$ & $80 \mathrm{PA} / 20 \mathrm{EL}$ & Thermetrics, Seattle, USA \\
\hline
\end{tabular}

$C O$ cotton, $E L$ elastane, $P E$ polyester, $P A$ polyamide fully developed sweating, and drying. Properties concerning the onset of sweating were evaluated by contact angle measurement (hydrophilicity), moisture management test (MMT_-wicking effect), and evaluation of water spreading on a vertical surface. For the fully developed sweating, which corresponds to the conditions for $R_{\mathrm{ET}}$ measurements, the maximal moisture content (MMC), skin thermal resistance, evaporative cooling efficiency, and the evaporative heat loss for two sweat rates were determined. The drying rate was measured to evaluate the drying properties of the fabrics. Additionally, a simulation of the human thermophysiological response with a head manikin coupled to a thermo-physiological model has been performed to evaluate the performance of the fabrics in a realistic scenario (Martínez et al. 2017).

For a given thermal sweating device, the surface area of the skin remains unchanged, while the mass of the dry skin differs between fabrics; therefore, the MMC and drying rate values have been related to the unit of surface area. In all measurements, the water was supplied to the back side of the fabrics, which complies with the direction of water supply to skins in thermal sweating devices. The fabric skins for measurements were prepared with a $10 \%$ stretch typically used in thermal sweating devices to ensure good contact.

Whenever the supply of water of a thermal device was used, the experiments were carried out using Nanopure water in order to prevent clogging of the sweating system. However, tap water was used in experiments with pre-wetted skin, similarly to the experimental protocol presented by Wang et al. (2010a, 2012, 2016) and Lu et al. (2016b).

\section{Contact angle measurement}

Three samples of $1 \times 1 \mathrm{~cm}$ of each fabric were conditioned for $12 \mathrm{~h}$ at air temperature $20 \pm 0.5^{\circ} \mathrm{C}$, relative humidity $65 \pm 5 \%$, and air speed $<0.1 \mathrm{~m} / \mathrm{s}$. Each sample was placed in a well plate insert (Fig. 1a), which provided a flat and slightly stretched fabric's surface. The measurements were performed using a contact angle measuring system (DSA25, Krüss $\mathrm{GmbH}$,
Germany) with dedicated software allowing the calculation of contact angles.

\section{Moisture management tests (MMT)}

Five circular samples ( $\Phi 80 \mathrm{~mm})$ of each fabric were conditioned for $12 \mathrm{~h}$ at air temperature $20 \pm 0.5^{\circ} \mathrm{C}$, relative humidity $65 \pm 5 \%$, and air speed $<0.1 \mathrm{~m} / \mathrm{s}$. The measurement principle has been described in the literature (Hu et al. 2005; Yao et al. 2006). The following parameters for the top and bottom surface of the fabrics were measured in a moisture management tester (M290MMT, SDL Atlas, USA): wetting time, maximum wetted radius, moisture spreading speed, and accumulative one-way transport capacity. The tests were performed with water supplied in the direction of the gravitational force.

\section{Water spreading on a vertical surface}

The MMT allows only testing samples placed horizontally, while most of the surface of thermal manikins is vertical; therefore, a visual analysis of water spreading was performed on the heated sweating cylinder Torso (Annaheim et al. 2015; Zimmerli and Weder 1997). A single sweating outlet was set to sweat for $60 \mathrm{~s}$ which resulted in $1.35 \pm 0.05 \mathrm{~g}$ of water delivered to the skin. Pictures of the wetted skin were made after $60 \mathrm{~s}$ of sweating and after additional $60 \mathrm{~s}$ without sweating. The wetted area and wetting ranges were calculated in a dedicated software (AutoCAD, Autodesk, USA) after manually redrawing the contours of the wet surfaces.

\section{Maximal moisture content per unit area (MMC)}

One sample of each fabric $(10 \times 10 \mathrm{~cm})$ was conditioned in dry state for $12 \mathrm{~h}$ at air temperature $21 \pm 0.7{ }^{\circ} \mathrm{C}$, relative humidity $70 \pm 5 \%$, and air speed $<0.1 \mathrm{~m} / \mathrm{s}$, pinned at the edges to avoid rolling of the fabric when wetted and weighted (Fig. 1b). The pinned samples were then submerged in tap water at room temperature for at least $1 \mathrm{~h}$ before the 
Fig. 1 a Sample for contact angle measurement. b Sample hung vertically with pins during MMC measurement. c MMC experimental setup. d Skin with foil covering taped to the heated guards of Torso for total thermal resistance measurement. e Skin for head manikin tests. f Sample on a horizontal wire net during drying rate measurement
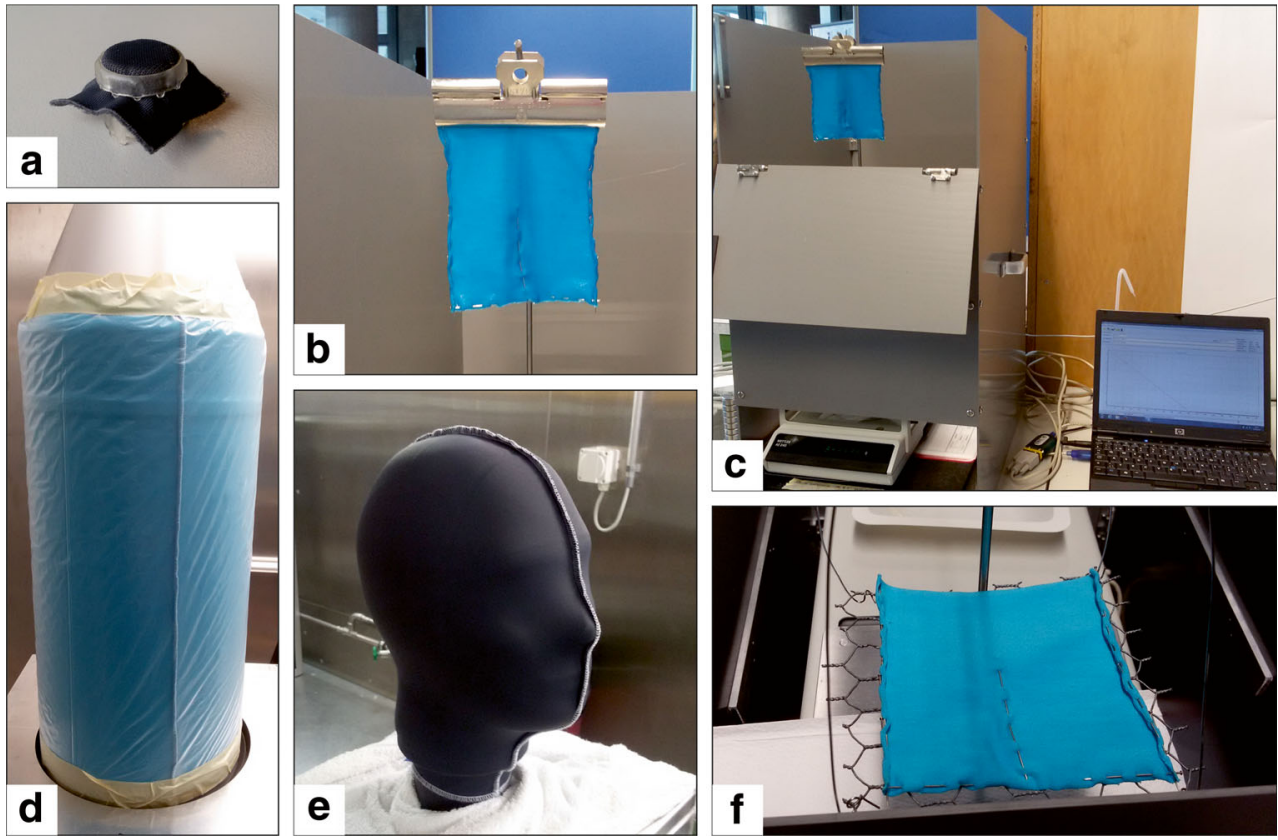

measurement. The experimental setup consisted of a stand with a hook placed on an analytical balance (Mettler AE240, Mettler-Toledo, Switzerland) with continuous recording of the mass loss (Fig. 1c). The sample was hung vertically with a clamp, and the whole setup was encased to impede the influence of air movement on the mass measurement. As the sample was soaked with water, droplets were forming on the lower end of the sample and were falling, which resulted in a decrease of the mass of the sample. The MMC was calculated based on the mass of the wet sample after the last droplet of water has been observed to drip, according to the following equation:

$\mathrm{MMC}=\frac{m_{\mathrm{wet}}-m_{\mathrm{dry}}}{A}\left(\frac{\mathrm{g}}{\mathrm{m}^{2}}\right)$,

where $m_{\text {wet }}$ and $m_{\text {dry }}$ are the mass of the sample in wet and dry state $(\mathrm{g})$, respectively, and $A$ is the surface area of the sample $\left(\mathrm{m}^{2}\right)$.

The measurements were repeated five times for each fabric and average MMC was calculated.

\section{Skin thermal resistance}

The thermal resistance properties of the fabric skins were measured on the heated sweating cylinder Torso in dry and wet conditions. The measurements were performed in a climate chamber at air temperature $20 \pm 0.5^{\circ} \mathrm{C}$, relative humidity $50 \pm 5 \%$, and air speed $<0.1 \mathrm{~m} / \mathrm{s}$, logged by microclimate sensors (Sensoanemo 5100CL, Sensors Electronic, Poland). The amount of tap water contained in the fabrics to achieve a fully saturated wet skin on Torso without dripping has been empirically assessed to be smaller than the MMC (i.e., 62, 63, 67 , and $87 \%$ of the MMC for $\mathrm{PE}, \mathrm{PA}, \mathrm{TH}$, and $\mathrm{CO}$, respectively). This was due to the stretching of the fabrics and the necessary manipulation of the skins while putting them on Torso. The wetted skins were sealed in plastic bags and conditioned in the climate chamber for $12 \mathrm{~h}$ to ensure homogenous moisture distribution in the fabrics. The surface temperature of Torso was set to $35^{\circ} \mathrm{C}$. Heat flux values were logged for $1 \mathrm{~h}$, and three repetitions were performed for each fabric in three states: dry skin only, dry skin with additional plastic foil, and wet skin with additional plastic foil, from which total thermal resistances $R_{\mathrm{dry}}, R_{\mathrm{dry}+\mathrm{foil}}$, and $R_{\text {wet+foil }}$ were calculated based on the mean heat flux value from 20 min steady-state period according to the following equation:

$R=\frac{\left(T_{\text {Torso }}-T_{\text {air }}\right)}{\mathrm{HF}}\left(\frac{\mathrm{m}^{2} \mathrm{~K}}{\mathrm{~W}}\right)$,

where $T_{\text {Torso }}$ is the mean surface temperature of Torso $\left({ }^{\circ} \mathrm{C}\right)$, $T_{\text {air }}$ is the mean air temperature $\left({ }^{\circ} \mathrm{C}\right)$, and $\mathrm{HF}$ is the mean heat flux on Torso $\left(\mathrm{W} / \mathrm{m}^{2}\right)$.

The plastic foil covering was taped to the heated guards to prevent evaporation of moisture during the wet skin measurements (Fig. 1d). In these conditions, the moisture can evaporate from the fabric and condense on the inner side of the foil, which leads to an increase of the foil temperature (Havenith et al. 2008). Therefore, thermal resistance determined in such conditions is called the apparent wet thermal resistance (Lotens et al. 1995; Wang et al. 2016). From the two measurements with dry skin, the thermal resistance of the foil $R_{\text {foil }}$ (Eq. 3) and then the apparent thermal resistance of the wet skin and the 
adjacent air layer without the foil $R_{\text {wet }}$ (Eq. 4) were calculated as follows:

$$
\begin{aligned}
& R_{\text {foil }}=R_{\text {dry }+ \text { foil }}-R_{\text {dry }}\left(\frac{\mathrm{m}^{2} \mathrm{~K}}{\mathrm{~W}}\right) \\
& R_{\text {wet }}=R_{\text {wet }+ \text { foil }}-R_{\text {foil }}\left(\frac{\mathrm{m}^{2} \mathrm{~K}}{\mathrm{~W}}\right)
\end{aligned}
$$

\section{Evaporative cooling efficiency}

The measurements were performed following the test protocol and equations by Havenith et al. (2008) in a climate chamber with air temperature equal to $34.1 \pm 0.2^{\circ} \mathrm{C}$, relative humidity equal to $18 \pm 5 \%$, i.e., water vapor pressure equal to $1 \mathrm{kPa}$, and air speed lower than $0.2 \mathrm{~m} / \mathrm{s}$. Each skin was wetted with tap water as described for wet skin thermal resistance measurements and conditioned in sealed bags in the climate chamber for at least $12 \mathrm{~h}$. The measurements were performed both on Torso and on a nine-zone head manikin (Martínez et al. 2017, Thermetrics, Seattle, USA; Fig. 1e) in the so-called isothermal conditions where $T_{\text {air }}=T_{\text {Torso }}=T_{\text {head manikin. }}$. After putting the wet skin on the thermal device, an immediate increase in the heat flux values was observed after which a steady-state measurement of evaporative heat loss was possible for at least 15 min before a sharp decrease in heat flux value was observed when the skin started to dry out. On the head manikin, it was not possible to obtain a steady-state with pre-wetted skin due to fast drying out of the upper zones related to gravitational moisture migration. Therefore, constant but individual flow set points for each zone have been empirically selected in a way that prevented skin drying out and any dripping and were as follows: forehead $310 \mathrm{ml} / \mathrm{m}^{2} \mathrm{~h}$, face $300 \mathrm{ml} / \mathrm{m}^{2} \mathrm{~h}$, left and right temple $420 \mathrm{ml} / \mathrm{m}^{2} \mathrm{~h}$ each, crown $330 \mathrm{ml} / \mathrm{m}^{2} \mathrm{~h}$, upper head $270 \mathrm{ml} / \mathrm{m}^{2} \mathrm{~h}$, lower head $220 \mathrm{ml} / \mathrm{m}^{2} \mathrm{~h}$, occipital $250 \mathrm{ml} / \mathrm{m}^{2} \mathrm{~h}$, and neck $200 \mathrm{ml} / \mathrm{m}^{2} \mathrm{~h}$, resulting in $0.66 \mathrm{~g} / \mathrm{min}$ of water delivered to the entire head.

Apart from heat flux measurements, also the mass loss rate was monitored by continuous weighing of the thermal device with the skin (Mettler-Toledo, Switzerland). For each fabric, three repetitions have been performed on Torso and five on the head manikin and mean values were calculated.

Since the measurements have been carried out in the socalled isothermal conditions (Havenith et al. 2008), the total real heat $\operatorname{loss} E_{\text {real }}$ observed was attributed to evaporation, i.e., no dry heat loss occurred. The evaporative cooling potential $E_{\text {mass }}$ has been calculated as the latent heat content of the water that was evaporating from the skin.

$E_{\text {mass }}=\dot{m} \times \lambda\left(\frac{\mathrm{W}}{\mathrm{m}^{2}}\right)$,

where $\dot{m}$ is the mass loss rate $\left(\mathrm{g} / \mathrm{m}^{2} \mathrm{~s}\right), \lambda$ is the enthalpy of evaporation, $\lambda=2420 \mathrm{~J} / \mathrm{g}$ (for $T_{\text {air }}=34{ }^{\circ} \mathrm{C}$ ).
The real evaporative cooling efficiency $\eta_{\text {real }}$ has been calculated according to the following equation:

$\eta_{\text {real }}=\frac{E_{\text {real }}}{E_{\text {mass }}} \times 100 \%$

\section{Evaporative heat loss on the head manikin}

The tests were performed in a climate chamber at air temperature $25 \pm 0.3{ }^{\circ} \mathrm{C}$, relative humidity $50 \pm 5 \%$, and air speed $<0.1 \mathrm{~m} / \mathrm{s}$, logged by microclimate sensors (Sensoanemo 5100CL, Sensors Electronic, Poland). The surface temperature of all head zones was set to $35^{\circ} \mathrm{C}$. The measurement for each skin consisted of five 1-h phases, such as dry phase, wet phase with sweat rate of $250 \mathrm{ml} / \mathrm{m}^{2} \mathrm{~h}(0.58 \mathrm{~g} / \mathrm{min}$ for the entire head), dry phase, and wet phase with sweat rate of $500 \mathrm{ml} / \mathrm{m}^{2} \mathrm{~h}(1.15 \mathrm{~g} / \mathrm{min}$ for the entire head). This alternation of dry and wet phases made it possible to obtain a steady-state at the end of each phase of the measurement for at least $15 \mathrm{~min}$. During the measurements, surface temperatures and heat fluxes for each zone were logged with the manikin's software (ThermDAC, Thermetrics, Seattle, USA). The measurements for each fabric were repeated three times, and the evaporative heat loss was calculated as the difference between average heat loss from steady-state in wet and dry conditions.

\section{Drying rate}

Samples for drying rate tests underwent the same conditioning process as for MMC tests and were performed on a slightly modified experimental setup with a wire net allowing horizontal hanging of the samples (Fig. 1f), which ensured homogenous drying of the entire surface of the fabric, in contrast to vertical hanging where the moisture gravitationally migrated to the lower end of the sample. The mass of the sample was recorded every $5 \mathrm{~s}$ until the fabric was dry, i.e., the mass was equal to the dry mass of the sample before wetting. Linear regression was applied to the recorded mass measurements to calculate the drying rate $\left(R^{2}>0.99\right)$. For each fabric, three repetitions were performed, from which the average value of drying rate was calculated.

\section{Simulation with the head manikin coupled to a thermo-physiological model}

In order to compare the performance of the fabric skins while simulating the human thermo-physiological response to an exposure, measurements were performed with a coupled system consisting of the head manikin and the model of human thermo-physiology by Fiala (Fiala and Havenith 2015, Martínez et al. 2016, Martínez et al. 2017, Psikuta et al. 2012; FPCm.5.3, Ergonsim, Germany). A real-time feedback 
loop allowed the head manikin to be controlled by the physiological model, where the model was providing local surface temperatures and sweat rates for the head according to the real heat loss from the manikin surface, and the remaining body parts were simulated purely virtually. The coupling has been described in detail by Martínez et al. (2017).

For this study, one experimental trial has been chosen, and coupled simulation tests were performed with all four skins in order to compare their reliability in reproducing experimental data from a human subject study. The experimental trial consisted of a pre-exercise standing phase (20 $\mathrm{min})$, a cycling phase (first 3 min with a metabolic rate of 2.5 met, then $45 \mathrm{~min}$ with $5.3 \mathrm{met}$ ), and a post-exercise standing phase (15 $\mathrm{min})$. The experimental conditions for the coupled simulation test have been described in detail by Martínez (2015, see Table 5.9 exposure 25). Three repetitions of the coupled simulation with each skin have been performed, and mean values of forehead temperature and sweat rate have been calculated.

Since the internal sweating system of the head manikin and the environmental conditions were the same in all measurements, the differences for forehead temperature and sweat rate between the four skins can be attributed to the different properties of the fabrics. To evaluate the performance of the skins, statistical indices such as root mean square deviation (rmsd) and bias of forehead temperature have been computed, according to the following equations (Psikuta et al. 2013):

$$
\begin{aligned}
& \text { rmsd }=\sqrt{\frac{\sum\left(t_{\text {measured }}-t_{\text {predicted }}\right)^{2}}{n}} \\
& \text { bias }=\frac{\sum\left(t_{\text {measured }}-t_{\text {predicted }}\right)}{n},
\end{aligned}
$$

where $t_{\text {measured }}$ is the measured forehead temperature from experiment, $t_{\text {predicted }}$ is the forehead temperature predicted by the coupled system, and $n$ is the number of experimental data points.

\section{Results}

The results for the measurements of contact angle, MMT, water spreading on a vertical surface, MMC, thermal resistance, evaporative cooling efficiency, drying rate, and evaporative heat loss from the head manikin are presented in Table 2. The water spreading on Torso is also presented in Fig. 2. The results from the simulations with the head manikin coupled to the thermo-physiological model are shown in Fig. 3 together with experimental values and pure physiological simulation values.

The measurement of contact angle for $\mathrm{CO}, \mathrm{PE}$, and $\mathrm{TH}$ was not possible, since the water was absorbed very fast and contact angle measurement was not possible to record by the measuring system. Therefore, these three fabrics can be assumed to be highly hydrophilic. The contact angle value for PA $\left(131.2^{\circ}\right)$ corresponds to a mostly non-wetting, hydrophobic fabric (de Gennes et al. 2004).

\section{Discussion}

\section{Onset of sweating}

Besides PA, the tested fabrics showed good performance in tests related to the onset of sweating. The hydrophilicity of $\mathrm{CO}, \mathrm{TH}$, and $\mathrm{PE}$ revealed in the contact angle measurement was confirmed in the MMT measurements with fast and very fast wetting times and moisture spreading speeds (Table 2). The values of bottom and top wetted radii which were assessed as large and very large, and high moisture spreading speed for these three fabrics are beneficial for the simulation of human-like sweating, i.e., when no pre-wetting is used. High standard deviation values from MMT tests of the PA fabric are related to the nonuniform wetting of the fabric due to its hydrophobicity which led to non-repeatable measurements, e.g., different shape and dimensions of the wetted area.

Since thermal manikins used with a fabric skin have a significantly inferior number of sweating outlets to human skin, good moisture spreading properties ensure a superficial wetting, as opposed to a more punctual liquid delivery if a hydrophobic fabric was used. Liquid spreading properties are of particular importance for the first phase of sweating as the cooling effect of sweating can only be fully measured once the moisture reaches the neighboring sensors embedded in the surface of the thermal device. If the wetted radius is too small, only negligible changes in heat loss will be measured, although some cooling will occur locally leading to inaccurate predictions from the physiological model during coupled simulations, which was reported by Psikuta et al. (2008) for the simulation of a warm exposure with low sweat rate.

The measured accumulative one-way transport capacity $R$ represents the ability of the fabric to wick moisture from the inner to the outer surface. The low value for $\mathrm{CO}$, i.e., $6 \%$ and the negative values for $\mathrm{PE}$ and $\mathrm{TH}$, i.e., -68 and $-70 \%$ (Table 2) show that the moisture stays partially near the heated surface of the sweating device, which might be a desirable property of skins for sweating simulations. It has been shown by Havenith et al. (2013) that the evaporative cooling efficiency decreases when the evaporation occurs farther away from the thermal device. Therefore, a poor accumulative one-way transport capacity might impede the migration of moisture towards clothing layers and as such provide more cooling at the surface of the manikin. However, in vertical orientation of the skin on the manikin, this effect might be lower than observed in this test, where the sample was oriented horizontally. 
Table 2 Summary of results (average value \pm standard deviation)

\begin{tabular}{|c|c|c|c|c|c|c|}
\hline & Test & Unit & $\mathrm{CO}$ & PE & $\mathrm{TH}$ & $\mathrm{PA}$ \\
\hline \multirow[t]{2}{*}{1} & \multirow[t]{2}{*}{ Contact angle } & Deg & - & - & - & $131.2 \pm 5.5$ \\
\hline & & Class & Hydrophilic & Hydrophilic & Hydrophilic & Hydrophobic \\
\hline \multirow{3}{*}{$\begin{array}{l}2 \\
2.1\end{array}$} & \multicolumn{6}{|l|}{ MMT } \\
\hline & $\mathrm{WT}_{\mathrm{T}}$ & $\mathrm{s}$ & $3.5 \pm 0.1$ & $2.8 \pm 0.1$ & $2.6 \pm 0.2$ & $20.3 \pm 20.9$ \\
\hline & & Class & Fast & Very fast & Very fast & Slow \\
\hline \multirow[t]{2}{*}{2.2} & $\mathrm{WT}_{\mathrm{B}}$ & $\mathrm{s}$ & $3.6 \pm 0.1$ & $3.0 \pm 0.1$ & $2.8 \pm 0.3$ & $8.9 \pm 1.5$ \\
\hline & & Class & Fast & Fast & Very fast & Medium \\
\hline \multirow[t]{2}{*}{2.3} & $\mathrm{MWR}_{\mathrm{T}}$ & $\mathrm{mm}$ & $20 \pm 0$ & $26 \pm 2$ & $30 \pm 0$ & $21 \pm 7$ \\
\hline & & Class & Large & Very large & Very large & Large \\
\hline \multirow[t]{2}{*}{2.4} & $\mathrm{MWR}_{\mathrm{B}}$ & $\mathrm{mm}$ & $20 \pm 0$ & $26 \pm 2$ & $30 \pm 0$ & $22 \pm 8$ \\
\hline & & Class & Large & Very large & Very large & Large \\
\hline \multirow[t]{2}{*}{2.5} & $\mathrm{SS}_{\mathrm{T}}$ & $\mathrm{mm} / \mathrm{s}$ & $3.2 \pm 0.1$ & $5.1 \pm 0.4$ & $7.0 \pm 0.8$ & $1.9 \pm 1.5$ \\
\hline & & Class & Fast & Very fast & Very fast & Slow \\
\hline \multirow[t]{2}{*}{2.6} & $\mathrm{SS}_{\mathrm{B}}$ & $\mathrm{mm} / \mathrm{s}$ & $3.1 \pm 0.1$ & $4.9 \pm 0.3$ & $6.9 \pm 0.8$ & $2.4 \pm 1.9$ \\
\hline & & Class & Fast & Very fast & Very fast & Medium \\
\hline \multirow[t]{2}{*}{2.7} & $R$ & $\%$ & $6 \pm 25$ & $-68 \pm 3$ & $-70 \pm 8$ & $115 \pm 147$ \\
\hline & & Class & Fair & Poor & Poor & Good \\
\hline 3 & \multicolumn{6}{|c|}{ Moisture spreading on a vertical surface } \\
\hline 3.1 & $\mathrm{WA}_{60}$ & $\mathrm{~cm}^{2}$ & 37.4 & 55.2 & 51.4 & 15.9 \\
\hline 3.2 & $\mathrm{WA}_{120}$ & $\mathrm{~cm}^{2}$ & 52.6 & 93.4 & 67.9 & 19.2 \\
\hline 3.3 & $\mathrm{WA}_{120} / \mathrm{WA}_{60}$ & - & 1.4 & 1.7 & 1.3 & 1.2 \\
\hline 3.4 & WR upwards & $\mathrm{cm}$ & 3.4 & 3.0 & 1.6 & 1.0 \\
\hline 3.5 & WR downwards & $\mathrm{cm}$ & 4.2 & 7.5 & 3.7 & 5.0 \\
\hline 3.6 & WR sideways $^{\mathrm{a}}$ & $\mathrm{cm}$ & 3.1 & 3.5 & 6.0 & 2.2 \\
\hline 4 & $\mathrm{MMC}$ & $\mathrm{g} / \mathrm{m}^{2}$ & $480 \pm 7$ & $485 \pm 12$ & $492 \pm 21$ & $577 \pm 2$ \\
\hline 5 & \multicolumn{6}{|l|}{ Isolative properties } \\
\hline 5.1 & $R_{\mathrm{dry}}$ & $\mathrm{m}^{2} \mathrm{~K} / \mathrm{W}$ & $0.109 \pm 0.002$ & $0.118 \pm 0.002$ & $0.103 \pm 0.001$ & $0.114 \pm 0.001$ \\
\hline 5.2 & $R_{\text {wet }}$ & $\mathrm{m}^{2} \mathrm{~K} / \mathrm{W}$ & $0.081 \pm 0.002$ & $0.089 \pm 0.004$ & $0.081 \pm 0.003$ & $0.085 \pm 0.006$ \\
\hline 5.3 & $R_{\text {wet }} / R_{\text {dry }}$ & $\%$ & $74.8 \pm 2.0$ & $75.3 \pm 3.1$ & $79.0 \pm 3.3$ & $75.1 \pm 5.9$ \\
\hline 6 & \multicolumn{6}{|c|}{ Evaporative cooling efficiency } \\
\hline 6.1 & Torso & $\%$ & $80.1 \pm 3.0$ & $87.4 \pm 0.3$ & $79.8 \pm 4.0$ & $86.1 \pm 1.9$ \\
\hline 6.2 & Head manikin & $\%$ & $80.1 \pm 3.4$ & $81.0 \pm 4.8$ & $81.3 \pm 2.8$ & $79.5 \pm 3.5$ \\
\hline 7 & Drying rate & $\mathrm{g} / \mathrm{m}^{2} \mathrm{~h}$ & $83.4 \pm 1.6$ & $82.6 \pm 0.9$ & $89.0 \pm 1.7$ & $83.6 \pm 1.2$ \\
\hline 8 & \multicolumn{6}{|c|}{ Evaporative heat loss head manikin } \\
\hline 8.1 & For $250 \mathrm{ml} / \mathrm{m}^{2} \mathrm{~h}$ & $\mathrm{~W} / \mathrm{m}^{2}$ & $138.5 \pm 25.4$ & $159.4 \pm 14.5$ & $158.1 \pm 20.4$ & $111.1 \pm 17.9$ \\
\hline 8.2 & For $500 \mathrm{ml} / \mathrm{m}^{2} \mathrm{~h}$ & $\mathrm{~W} / \mathrm{m}^{2}$ & $216.6 \pm 1.6$ & $202.8 \pm 3.1$ & $222.1 \pm 4.8$ & $105.5 \pm 9.5$ \\
\hline
\end{tabular}

$C O$ cotton with elastane, $P E$ polyester, $T H$ Thermetrics' skin, $P A$ polyamide with elastane, $M M T$ moisture management test, class classification, $W T_{T}, W T_{B}$ wetting time of top and bottom surface, $M W R_{T}, M W R_{B}$ maximum wetted radius of top and bottom surface, $S S_{T}, S S_{B}$ moisture spreading speed of top and bottom surface, $R$ accumulative one-way transport capacity, $W A_{60}, W A_{120}$ wetted area after 60 and $120 \mathrm{~s}, W R$ wetting range, $M M C$ maximal moisture capacity per area, $R_{d r v}, R_{\text {wet }}$ total thermal resistance of the skin with adjacent air layer in dry and wet conditions

${ }^{\text {a }}$ Mean value from the left and right WR
Thermal sweating devices, such as cylinders and body-part or full-body manikins, have mostly vertical surfaces; therefore, the measurements on Torso provided valuable information on the vertical moisture spreading. Poor liquid spreading in the PA skin was confirmed; the water supplied to the fabric was passing through it and rolling off without wetting the skin
(Fig. 2d). For CO, PE, and TH, the wetted area had noticeably different shapes, although all three fabrics showed similar performance during MMT measurements (Fig. 2a-c). This indicates that the skin and the thermal sweating manikin should match each other, i.e., the skin properties ought to ensure enough water spreading for a specified number and location 
Fig. 2 Moisture spreading on a vertical surface after $60 \mathrm{~s}$ with wetting and additional $60 \mathrm{~s}$ without wetting for fabrics a cotton $\mathrm{CO}, \mathbf{b}$ polyester PE, $\mathbf{c}$ Thermetrics' skin TH, and d polyamide PA
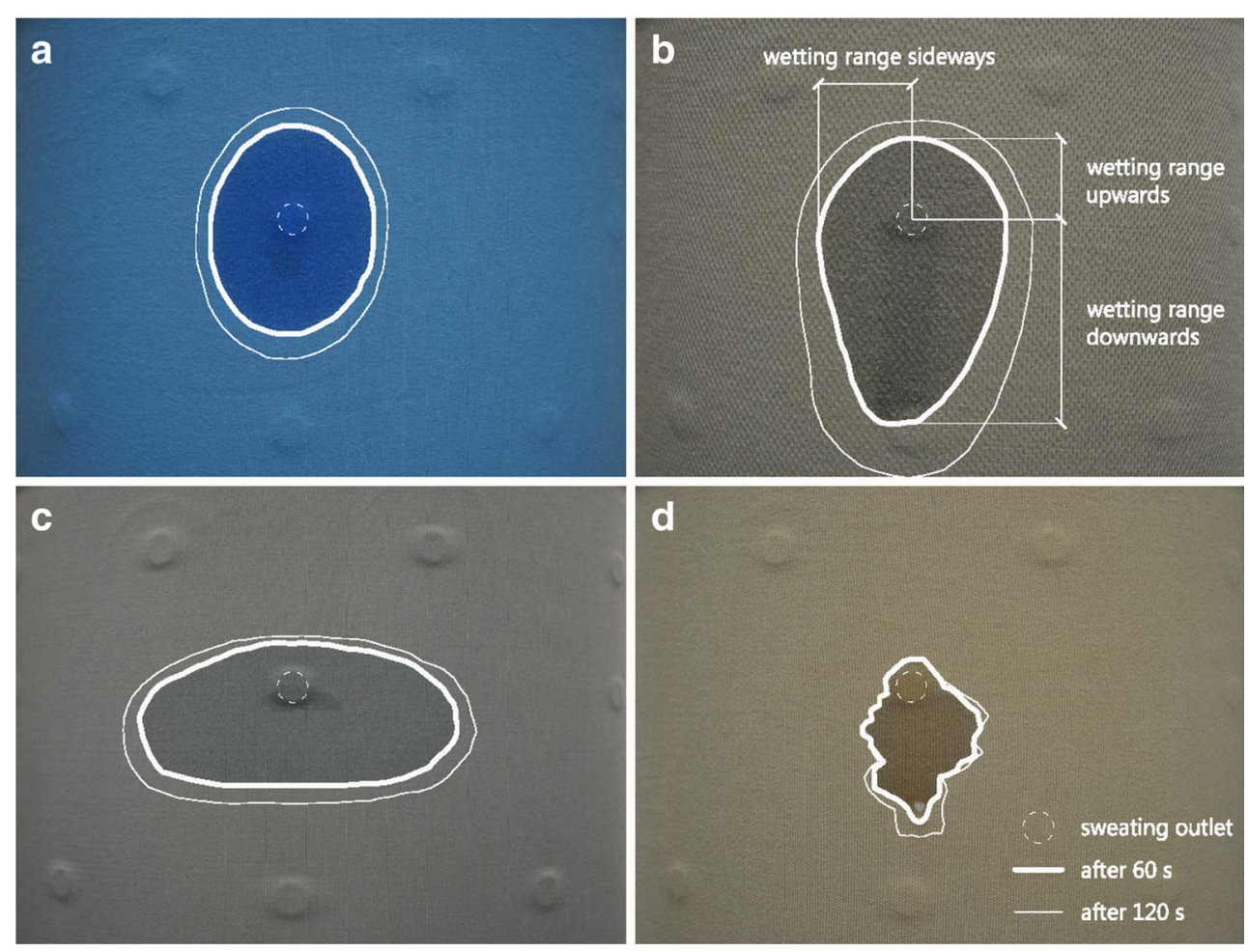

of sweating outlets. For example, for an efficient wetting of the TH skin, the horizontal distance between outlets can be much bigger due to a higher lateral wetting range, but the vertical distance should be smaller than for the other tested fabrics (Table 2, Fig. 2). To avoid moisture migration and dripping of water, fabrics with lower downward wetting range should be chosen for $R_{\mathrm{ET}}$ measurements, i.e., $\mathrm{CO}$ or $\mathrm{TH}$ (Table 2).

The contact angle and moisture spreading on a vertical surface evaluation can be recommended as valuable measurements for the evaluation of fabrics' properties related to the initial phase of sweating. Checking the hydrophilicity of any fabric by measuring the contact angle is a fast and efficient way of determining whether a fabric could be suitable for use as a skin. The example of $\mathrm{TH}$ and PA fabrics, which had utterly different wetting properties despite a similar fabric's composition (Table 1), shows that it is not possible to evaluate the properties of a skin based only on its fiber content. Fabric tightness (Yanilmaz and Kalaoğlu 2012) and finishing treatment play also an important role and their resultant effect can
Fig. 3 Forehead temperature and flow set point: experimental data from exposure, values from virtual simulation, i.e., with physiological model only, and from coupled simulations with all four skins; for forehead temperature, the rmsd and bias values are presented
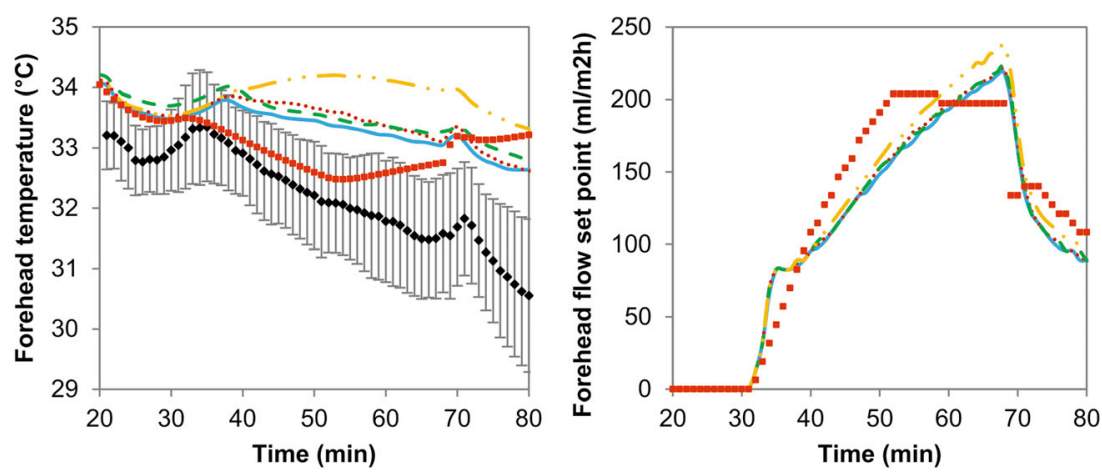

\begin{tabular}{lcc}
\hline & rmsd & bias \\
\hline Virtual & 1.11 & -0.87 \\
\hline TH & 1.23 & -1.15 \\
CO & 1.35 & -1.26 \\
PE & 1.40 & -1.33 \\
PA & 1.83 & -1.66 \\
\hline
\end{tabular}

- Experimental data

- Virtual

- TH

........ CO

- - PE

-.. PA 
be summarized by contact angle measurement. For the water spreading properties of fabrics, the evaluation on a vertical setup provides more valuable and realistic information for manikins' skins, since the manikins' surfaces are mostly vertical.

\section{Fully developed sweating}

In the case of simulation of thermo-physiological responses, the MMC value can be interpreted only for fabrics with good moisture spreading properties, since the full wetting of the skin is otherwise unlikely, e.g., in the case of PA skin the MMC equal to $577 \mathrm{~g} / \mathrm{m}^{2}$ may not be possible to reach without pre-wetting of the fabric (Table 2). Berglund (1971) calculated the critical thickness of water layer on the human body above which dripping occurs to be $37.6 \mu \mathrm{m}$, i.e., $37.6 \mathrm{~g} / \mathrm{m}^{2}$ of body surface. However, data were collected only from one subject. Also, the water trapped inside the fabric does not behave in the same way as sweat in the form of droplets on the human skin and, therefore, the critical thickness of water layer given by Berglund (1971) cannot be directly compared to the MMC values for the tested fabrics.

It has been shown by Wang et al. (2016) that the more moisture added to clothing, the smaller the apparent wet thermal insulation. Therefore, the skin thermal resistance during thermo-physiological simulation with a thermal manikin can vary between its maximal dry value and the wet skin insulation value. For the tested fabrics, the apparent thermal resistance of wet skins decreased by $25 \%$ for $\mathrm{CO}, \mathrm{PE}$, and PA and by $21 \%$ for $\mathrm{TH}$ in comparison to the value for dry skin (Table 2). The knowledge about thermal resistance of the fabrics in both dry and wet state may contribute to the development of coefficients to include in thermo-physiological model's predictions, in order to take into account the additional insulation resulting from the skin while carrying out coupled simulations, especially for low sweat rates.

The evaporative cooling efficiency measurements showed differences between the evaporative heat loss determined by the heat loss and mass loss methods for all four tested fabrics (Table 2). As described in the literature (Burton 1944; Wang et al. 2011), some of the heat for evaporation is taken from the environment, leading to the discrepancies between the two calculation methods. In the measurement performed on Torso, PA and PE skins showed a higher evaporative cooling efficiency than $\mathrm{CO}$ and $\mathrm{TH}$ fabrics, i.e., 86 and $87 \%$ for PA and $\mathrm{PE}$ and 80 and $82 \%$ for $\mathrm{CO}$ and TH (Table 2). However, the measurements performed on the head manikin led to similar values for all four tested skins, i.e., approximately $80 \%$ of evaporation heat taken from the head manikin and $20 \%$ from the environment (Table 2). The evaporative cooling efficiency values obtained from measurements on the head manikin are comparable with the one presented by Havenith et al. (2008) for an impermeable ensemble, that is approximately $80-85 \%$ and by $\mathrm{Lu}$ et al. (2016a) for a wet skin only, which equaled $85 \%$. The results from Wang et al. (2011) for a wet skin only were lower, i.e., $73 \%$. It has to be noted, however, that the measurement conditions were not the same in all of these studies. The reason for the observed discrepancies in evaporative cooling efficiency values on Torso and on the head manikin for two fabrics, i.e., PE and PA, may be due to different sweating simulation methods, i.e., pre-wetting of the skin for Torso measurements and pre-wetting with additional constant moisture delivery to the skin for the measurements on the head manikin. When no additional water is supplied to the skin during the test, the real water vapor pressure at the skin surface may differ from the one assumed for fully saturated fabric, which was described by Lu et al. (2016b). These discrepancies may have been more pronounced in the case of PE and PA skins due to the fabrics' individual properties. Additionally, the shape of Torso allowed a full contact of the skin with the device, whereas less contact was ensured on the head manikin due to the anatomical shape of the device.

The mean evaporative heat loss on the head manikin for a low flow set point, i.e., $250 \mathrm{ml} / \mathrm{m}^{2} \mathrm{~h}$, had a high standard deviation for all tested fabrics, corresponding to high values of the coefficient of variation, i.e., ranging from $9 \%$ for PE to $18 \%$ for CO (Table 2). The lower repeatability of these measurements might have been caused by the fact that only some of the sensors embedded in the manikin surface could detect the increased heat loss due to evaporative heat and even small differences in the wetted area shape between the repetitions could affect the measured values. The evaporative heat loss measurements for a sweat rate of $500 \mathrm{ml} / \mathrm{m}^{2} \mathrm{~h}$ had higher repeatability for the individual fabrics (Table 2). The low and comparable values of evaporative heat loss for both flow set points in the case of the PA skin, i.e., 111.1 and $105.5 \mathrm{~W} /$ $\mathrm{m}^{2}$, are a consequence of the hydrophobicity of the fabric which caused a limited wetting of the skin.

For the evaluation of fabrics or the development of new fabrics for use as a sweating skin, the measurements of MMC, cooling evaporative resistance, and isolative properties are suggested as relevant tests of the properties related to the fully developed phase of sweating.

\section{Drying phase}

For simulations with lower sweat rates, i.e., when all the supplied water would stay trapped inside the skin and no dripping would occur, the drying rate provides direct information about the drying time of each fabric. However, the drying time of fully wetted skins depends also on their MMC, i.e., the mass amount of water to evaporate varies between fabrics. For instance, the drying rates of $\mathrm{CO}$ and PA were found to be very similar, i.e., 83.4 and $83.6 \mathrm{~g} / \mathrm{m}^{2} \mathrm{~h}$, but when fully wetted the CO skin would dry faster due to a $23 \%$ smaller MMC than the PA fabric (Table 2). 
In the case of standard $R_{\mathrm{ET}}$ measurements with a prewetted skin, a combination of high MMC and low drying rate is beneficial to obtain longer steady-state heat flux measurement. Therefore, the PA fabric has better drying properties for $R_{\mathrm{ET}}$ measurement than the other three fabrics (Table 2). This advantage only applies for pre-wetted skin as the water spreading properties of the PA skin have been found to be poor.

\section{Coupled simulation with wetting and drying phases}

The coupled simulation performed with the head manikin controlled by the thermo-physiological model permitted to evaluate the suitability of the fabrics for simulating a realistic sweating scenario. The accuracy of the coupled simulation in reproducing the forehead temperature was influenced by three factors, namely, the accuracy of the pure virtual simulation, the environmental conditions during the exposure, and the properties of the fabric skin. During the coupled simulation, the thermo-physiological model and the environmental conditions were not modified, with the skins being the only changing factor. It can be seen in Fig. 3 that the use of CO, PE, and TH skin led to similar forehead temperature and sweat rate distribution for the simulated scenario. The inadequacy of PA for use as a skin demonstrated in the basic tests was confirmed by the higher rmsd value and the higher temperature and sweat rate predictions. However, more examples of such coupled simulations could confirm the good performance of the $\mathrm{CO}$, PE, and TH skins.

In the pure virtual simulation, it is assumed that $100 \%$ of latent heat is taken from the body surface. However, the cooling efficiency tests showed that around $80 \%$ of heat was taken from the surface of the head manikin, with the remaining $20 \%$ being taken from the surrounding environment (Table 2). This is why the forehead temperature was higher for the coupled simulation than in the case of pure virtual simulation in the developed phase of sweating (Fig. 3, after approx. $40 \mathrm{~min}$ ).

\section{Conclusions}

The properties of fabrics to be used as a skin for thermal sweating devices such as manikins have been investigated in basic tests with respect to three phases of sweating, namely, the onset of sweating, fully developed sweating, and drying phase. Overall, the $\mathrm{CO}, \mathrm{PE}$, and $\mathrm{TH}$ fabrics showed a similarly good performance related to tests with a thermo-physiological human simulator and can be, therefore, recommended for use with thermal sweating manikins. However, the fabric should always be chosen to match the sweating system of the manikin itself, e.g., the spacing between sweating outlets, to ensure that the moisture spreading properties will allow for efficient skin wetting. The $\mathrm{CO}$ fabric showed the best properties for $R_{\mathrm{ET}}$ measurements, i.e., low downward wetting range and desired combination of MMC and drying rate. Also, the use of PA for $R_{\mathrm{ET}}$ measurements with pre-wetted skin could be considered due to its high MMC and low drying rate, although the poor wetting properties of this fabric hinder its use in measurement with gradual sweating.

The study shows the different requirements for skins for $R_{\mathrm{ET}}$ measurements and for the simulation of human thermophysiological responses. The presented tests form a methodology for comparing different fabrics to be used as a sweating skin. Moreover, the discussion of various properties of skins can be useful for the development of new fabrics suitable for sweating simulations.

Acknowledgements We would like to express our gratitude to Natividad Martínez for all her kind help with the measurements on the head manikin and Emel Mert for the discussions about fabrics properties. We thank Thermetrics for providing samples of their fabric for the measurements.

\section{Compliance with ethical standards}

Conflict of interest The authors declare that they have no conflict of interest.

\section{References}

Annaheim S, Wang L-c, Psikuta A, Morrissey MP, Camenzind MA, Rossi RM (2015) A new method to assess the influence of textiles properties on human thermophysiology. Part I: thermal resistance. International Journal of Clothing Science and Technology 27:272282. doi:10.1108/IJCST-02-2014-0020

ASTM (2015) ASTM F2370 standard test method for measuring the evaporative resistance of clothing using a sweating manikin. American Society for Testing and Materials, Philadelphia, PA

Berglund LG (1971) A theoretical and experimental study of the thermoregulatory effects of salt accumulating on human skin during sweating. Dissertation, Kansas State University

Burke R, McGuffin R (2001) Development of an advanced thermal manikin for vehicle climate evaluation. In: Proceedings of 4th International Meeting on Thermal Manikins, 2001. pp 14-18

Burton AC (1944) An analysis of the physiological effects of clothing in hot environments. Report of Associate Committee on Aviation Medical Research C2754

de Gennes P-G, Brochard-Wyart F, Quéré D (2004) Capillarity and wetting phenomena: drops, bubbles, pearls, waves. Springer Science \& Business Media

Fan J, Chen Y (2002) Measurement of clothing thermal insulation and moisture vapour resistance using a novel perspiring fabric thermal manikin. Meas Sci Technol 13:1115. doi:10.1088/0957-0233/13/7/ 320

Fiala D, Havenith G (2015) Modelling human heat transfer and temperature regulation. In: The mechanobiology and mechanophysiology of military-related injuries. Springer, 265-302

Foda E, Siren K (2012) A thermal manikin with human thermoregulatory control: Implementation and validation. Int J Biometeorol 56:959971 
Havenith G, Bröde P, den Hartog E, Kuklane K, Holmér I, Rossi RM, Richards M, Farnworth B, Wang X (2013) Evaporative cooling: effective latent heat of evaporation in relation to evaporation distance from the skin. J Appl Physiol 114:778-785. doi:10.1152/ japplphysiol.01271.2012

Havenith G, Richards M, Wang X, Bröde P, Candas V, den Hartog E, Holmér I, Kuklane K, Meinander H, Nocker W (2008) Apparent latent heat of evaporation from clothing: attenuation and "heat pipe" effects. J Appl Physiol 104:142-149. doi:10.1152/japplphysiol. 00612.2007

Hu J, Li Y, Yeung K-W, Wong AS, Xu W (2005) Moisture management tester: a method to characterize fabric liquid moisture management properties. Text Res J 75:57-62. doi:10.1177/004051750507500111

ISO (1993) Textiles - physiological effects - measurement of thermal and water vapour resistance under steady-state conditions (sweating guarded hot plate test). International Organization for Standardization, Geneva, Switzerland (ISO 11092:1993)

Kuklane K, Heidmets S, Johansson T (2006) Improving thermal comfort in an orthopaedic aid: better Boston brace for scoliosis patients. In: 6th International Thermal Manikin and Modeling Meeting (6I3M), 2006. Hong Kong Polytechnic University, pp 343-351

Lotens W, Van De Linde F, Havenith G (1995) Effects of condensation in clothing on heat transfer. Ergonomics 38:1114-1131

Lu Y, Wang F, Peng H, Shi W, Song G (2016a) Effect of sweating set rate on clothing real evaporative resistance determined on a sweating thermal manikin in a so-called isothermal condition $(\mathrm{T}$ manikin $=$ $\mathrm{T} \mathrm{a}=\mathrm{T} \mathrm{r}$ ). Int J Biometeorol 60:481-488. doi:10.1007/s00484-0151029-3

Lu Y, Wang F, Peng H (2016b) Effect of two sweating simulation methods on clothing evaporative resistance in a so-called isothermal condition. Int J Biometeorol 60:1041-1049. doi:10.1007/s00484015-1095-6

Martínez N (2015) Multi-sector thermophysiological head simulator for headgear research. Dissertation, Universitat Politècnica de València

Martínez N, Psikuta A, Corberán JM, Annaheim S, Rossi RM (2017) Multi-sector thermophysiological head simulator for headgear research. Int J Biometeorol 61:273-285. doi:10.1007/s00484-0161209-9

Martínez N, Psikuta A, Kuklane K et al (2016) Validation of the thermophysiological model by Fiala for prediction of local skin temperatures. Int J Biometeorol 60:1969-1982. doi:10.1007/ s00484-016-1184-1

Meinander H (1997) Experience with a sweating thermal manikin — ready for standard use? In: Proceedings of a European seminar on thermal manikin testing. National Institute for Working Life, Stockholm, Sweden, 1997. pp 38-42

Psikuta A, Fiala D, Laschewski G, Jendritzky G, Richards M, Błażejczyk K, Mekjavič I, Rintamäki H, de Dear R, Havenith G (2012) Validation of the Fiala multi-node thermophysiological model for UTCI application. Int J Biometeorol 56:443-460. doi:10.1007/ s00484-011-0450-5

Psikuta A, Kuklane K, Bogdan A, Havenith G, Annaheim S, Rossi RM (2016) Opportunities and constraints of presently used thermal manikins for thermo-physiological simulation of the human body. Int $\mathbf{J}$ Biometeorol 60:435-446

Psikuta A, Richards M, Fiala D (2008) Single-sector thermophysiological human simulator. Physiol Meas 29:181-192. doi:10.1088/0967$3334 / 29 / 2 / 002$
Psikuta A, Wang L-C, Rossi RM (2013) Prediction of the physiological response of humans wearing protective clothing using a thermophysiological human simulator. J Occup Environ Hyg 10: 222-232. doi:10.1080/15459624.2013.766562

Psikuta A, Hepokoski M, Burke R, Schwenn T, Rossi R (2014) A methodology for the validation of thermophysiology models and adaptive manikins. 10th International Meeting on Thermal Manikin and Modelling (10i3m). Tampere, Finland

Richards M, Mattle N (2001) Development of a sweating agile thermal manikin (SAM). In: Proceedings of the 4th International Meeting on Thermal Manikins, 2001

Rugh JP, Farrington RB, Bharathan D, Vlahinos A, Burke R, Huizenga C, Zhang $H$ (2004) Predicting human thermal comfort in a transient nonuniform thermal environment. Eur J Appl Physiol 92:721-727

Ueno S, S-i S (2012) Correction of the evaporative resistance of clothing by the temperature of skin fabric on a sweating and walking thermal manikin. Text Res J 82:1143-1156. doi:10.1177/ 0040517511427966

Wang F-M, Gao C-S, Kuklane K, Holmér I (2009) A study on evaporative resistances of two skins designed for thermal manikin Tore under different environmental conditions. Journal of Fiber Bioengineering and Informatics 1:301-306. doi:10.3993/ jfbi03200908

Wang F-M, Kuklane K, Gao C-S, Holmér I, Havenith G (2010a) Development and validation of an empirical equation to predict wet fabric skin surface temperature of thermal manikins. Journal of Fiber Bioengineering and Informatics 3:9-15. doi:10.3993/ jfbi06201002

Wang F, Gao C, Kuklane K, Holmér I (2011) Determination of clothing evaporative resistance on a sweating thermal manikin in an isothermal condition: heat loss method or mass loss method? Ann Occup Hyg 55:775-783. doi:10.1093/annhyg/mer034

Wang F, Kuklane K, Gao C, Holmer I (2012) Effect of temperature difference between manikin and wet fabric skin surfaces on clothing evaporative resistance: how much error is there? Int J Biometeorol 56:177-182. doi:10.1007/s00484-011-0411-z

Wang F, Kuklane K, Gao C, Holmér I (2010b) Development and validity of a universal empirical equation to predict skin surface temperature on thermal manikins. J Therm Biol 35:197-203. doi:10.1016/j. jtherbio.2010.03.004

Wang L, Lu M, Dai X (2015) A study on material of manikin skin for measuring clothing evaporative resistance. Journal of Fiber Bioengineering and Informatics 8:221-228. doi:10.3993/ jfbim00088

Wang F, Shi W, Lu Y, Song G, Rossi RM (2016) Effects of moisture content and clothing fit on clothing apparent 'wet' thermal insulation: a thermal manikin study. Text Res J 86:57-63. doi:10.1177/ 0040517515580527

Yanilmaz M, Kalaoğlu F (2012) Investigation of wicking, wetting and drying properties of acrylic knitted fabrics. Text Res J 82(8):820 831. doi:10.1177/0040517511435851

Yao B-G, Li Y, Hu J-y, Y-1 K, K-w Y (2006) An improved test method for characterizing the dynamic liquid moisture transfer in porous polymeric materials. Polym Test 25:677-689. doi:10.1016/j. polymertesting.2006.03.014

Zimmerli T, Weder MS (1997) Protection and comfort—a sweating torso for the simultaneous measurement of protective and comfort properties of PPE. In: Performance of protective clothing: Sixth Volume, 1997. ASTM International, pp 271-280 